\title{
Sex, gender and emerging infectious disease surveillance: a leptospirosis case study
}

\author{
Jozica Skufca a and Yuzo Arima ${ }^{a}$ \\ Correspondence to Yuzo Arima (e-mail: arimay@wpro.who.int).
}

W hatever the population, wherever the place, whenever the period, sex has been an essential demographic variable for surveillance. However, the distinction between "sex" and "gender" has not always been well understood or acknowledged by those of us engaged in public health surveillance. Sex refers to the biological and physiological factors that define males and females, while gender refers to socially constructed roles and attributes that a particular society considers appropriate for men and women. ${ }^{1}$ While both sex and gender factors contribute to reported surveillance data, their full contributions are often not recognized. When such data are then used to ascertain sex/gender differential in disease risk without caution, the complete picture behind the observed distribution may be missed or misinterpreted. Using leptospirosis as a case example, we describe the importance of interpreting surveillance data with a more gender-sensitive perspective, considering the various biological and social factors behind the reported numbers.

Leptospirosis is an emerging infectious disease with a high public health burden in the Asia Pacific region. Human infection is caused by the Leptospira bacteria and usually occurs through exposure to urine of an infected animal, contaminated water or soil. A commonly cited risk factor for the disease is male sex/gender, ${ }^{2}$ and an excess of male leptospirosis cases observed in surveillance data is often ascribed to occupational/recreational exposures associated with male gender. However, it is often unknown how this observed distribution may be affected by sex differentials in disease severity or gender differentials in health care-seeking behaviour/access (Figure 1). These factors should be carefully considered when interpreting surveillance data.

Figure 1. Factors to consider when interpreting the observed sex distributions in reported surveillance data

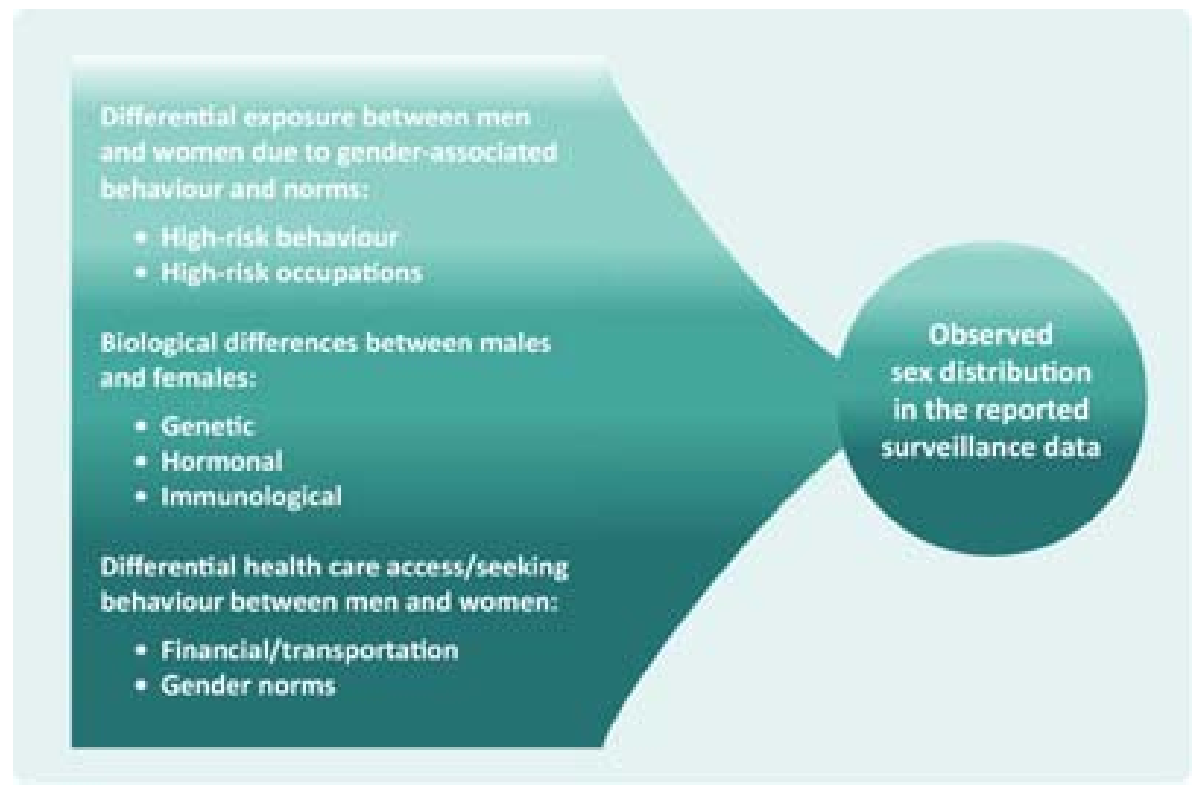

Emerging Disease Surveillance and Response, Division of Health Security and Emergencies, Western Pacific Regional Office, World Health Organization, Manila, Philippines. 
Traditionally, the excess of male leptospirosis cases observed in surveillance data has been explained through occupational/recreational exposures that put men in greater contact with leptospira-infected animals or contaminated water. ${ }^{1,3-5}$ For example, in the leptospirosis-endemic Philippines, from 1998 to 2001, among 840 clinically suspected seropositive cases, $87 \%$ of the cases were male and $70 \%$ were involved in outdoor activities, with $80 \%$ exposed to surface water or sewage. While there has been a considerable decrease in leptospirosis cases in Japan (attributed to occupational exposure control measures among rice-field workers), there continues to be an excess of male cases (16/20 cases reported from November 2003 to April 2005 were male), with the majority linked to maledominated occupations (e.g. sewage work). ${ }^{5}$ Similar findings have been reported from New Zealand, where $774 / 878$ (88\%) reported cases from 1999 to 2008 were male and $72 \%$ of the cases were livestock workers or meat-processing workers. ${ }^{3}$

Recently, biological differences have been cited as a possible, alternative factor for the male excess in reported leptospirosis cases. ${ }^{6,7}$ Several European studies have found that, while the incidence of leptospirosis is higher in men, there is no sex difference in leptospirosis seroprevalence, indicating that there may be sex differentials in the clinical manifestation of leptospirosis. ${ }^{4,7}$ Indeed, a recent study from Germany found that male leptospirosis patients $(n=263)$, relative to their female counterparts $(n=75)$, had clinically more severe outcomes and higher case fatality ( $5 \%$ versus $1 \%$, respectively) despite no significant differences in the type of exposure or time from onset of symptoms to treatment (4.5 days for both). ${ }^{7}$ In rural Lao People's Democratic Republic, while the proportion of persons engaged in agricultural work was found to be equal among males and females, leptospirosis seroprevalence was significantly higher among males (29\%) than females (19\%). When adjusted for previously reported risk factors for leptospirosis, such as barefoot walking and swimming, males were still significantly associated with higher seropositivity. ${ }^{8}$ In fact, there has been a growing recognition that biological differences between males and females based on genetic, immunological and hormonal factors may determine the susceptibility to disease and clinical outcomes. ${ }^{9}$ It is possible that females, when infected by leptospirosis (and all other things being equal), may have less severe sequalae than males, and surveillance data may be capturing the more severe cases. $^{7}$ In addition, a study conducted among leptospirosis patients in Sri Lanka revealed that male cases had significantly higher levels of leptospiremia than female cases (150 640 versus 5611 leptospira/mL, respectively). ${ }^{6}$

However, whether higher case numbers in males are due to a differential physiological response or to differentials in the dose received at the time of exposure, remains difficult to determine. Even when both genders are exposed to occupational/recreational risk factors, males may be receiving a higher dose due to greater frequency and/or duration of exposure. For instance, a study in Italy hypothesized that, among men and women both exposed to animals and/or leisure activities, the higher seropositivity among men (49/107; 46\%) relative to women $(47 / 180 ; 26 \%)$ may have been due to women being more cautious when in contact with animals or at leisure, thus receiving a lower dose. ${ }^{4}$ Being familiar with such gender-associated norms or behaviours of the population under surveillance is thus important, given the limitations in exposure measurement.

Whether the observed male excess in leptospirosis surveillance data is due to differential exposure, severity or both remains debatable, but both point to male gender as the higher risk group. However, gender differences in health care access or health care-seeking behaviour should also be considered. As surveillance data reflect only those cases that seek health care, any difference between the genders in health care accessibility (e.g. men have better access due to transportation or for financial reasons) or health care-seeking behaviour (e.g. men seek health care more often than women) would directly affect the surveillance data. For example, while there is an excess of male leptospirosis cases reported from India, a pattern traditionally linked to their greater occupational exposure, ${ }^{10}$ India is also among the lowest ranked nations in terms of gender equity. ${ }^{11}$ As some Indian women have a lack of education and the financial means to access and use health care, ${ }^{11}$ female leptospirosis cases may be underestimated in these settings. For example, among 143 patients affected by a leptospirosis outbreak in Orissa, India, while men compared to women had a higher attack rate $(6.8 \%$ versus $4.9 \%$, respectively) and proportion of cases hospitalized $(50 \%$ versus $40 \%$, respectively), the case fatality rate was significantly lower in males than in females ( $2 \%$ versus $16 \%$, respectively). ${ }^{12}$ Conversely, in other settings, there may be lower health 
care-seeking behaviour by men, such that the incidence among men may be underestimated. ${ }^{13}$

Interpretations based on observed sex/gender distributions from surveillance data require careful thought, as there are important implications for public health actions. If the male excess in leptospirosis cases can be validly attributed to their occupational/ recreational exposures, focusing public health efforts on reducing those exposures would be important. If, on the other hand, males have a more severe clinical outcome post-infection, emphasizing early and proper treatment for men might be important. However, if access to health care by women is known to be a concern for the population, one should interpret the reported sex distribution being mindful of such context. We hope that adopting a more gender-sensitive approach will assist all of us in public health practice to interpret surveillance data thoughtfully and to be mindful of the possible gender-related context of the reported numbers. Public health responses that follow such careful interpretation could enhance the efficiency and effectiveness of our actions.

\section{Conflicts of Interest}

None declared.

\section{Funding}

This study was undertaken as part of routine activities of the World Health Organization Regional Office for the Western Pacific.

\section{Acknowledgements}

The authors would like to thank Dr Tamano Matsui for reviewing the manuscript.

\section{References:}

1. Taking sex and gender into account in emerging infectious disease programmes: an analytical framework. Manila, World Health Organization, Regional Office for the Western Pacific, 2011.

2. Victoriano AFB et al. Leptospirosis in the Asia Pacific region. BMC Infectious Diseases, 2009, 9:147. doi:10.1186/14712334-9-147 pmid:19732423

3. Paine S. Bound Volume for the degree of Masters of Applied Epidemiology [Masters thesis]. Canberra, Australian National University, 2011.

4. Cacciapuoti B et al. Survey on the prevalence of leptospira infections in the Italian population. European Journal of Epidemiology, 1994, 10:173-180. doi:10.1007/BF01730367 pmid:7813695

5. Yanagihara $Y$ et al. Current status of leptospirosis in Japan and Philippines. Comparative Immunology, Microbiology and Infectious Diseases, 2007, 30:399-413. doi:10.1016/j. cimid.2007.05.003 pmid:17614131

6. Agampodi SB et al. Utility of quantitative polymerase chain reaction in leptospirosis diagnosis: association of level of leptospiremia and clinical manifestations in Sri Lanka. Clinical Infectious Diseases, 2012, 54:1249-1255. doi:10.1093/cid/ cis035 pmid:22354922

7. Jansen A et al. Sex differences in clinical leptospirosis in Germany: 1997-2005. Clinical Infectious Diseases, 2007, 44:69-72. doi:10.1086/513431 pmid:17407027

8. Kawaguchi $L$ et al. Seroprevalence of leptospirosis and risk factor analysis in flood-prone rural areas in Lao PDR. The American Journal of Tropical Medicine and Hygiene, 2008, 78:957-961. pmid: 18541776

9. Ober C, Loisel DA, Gilad Y. Sex-specific genetic architecture of human disease. Nature Reviews. Genetics, 2008, 9:911-922. doi:10.1038/nrg2415 pmid:19002143

10. Guidelines for Prevention and Control of Leptospirosis. Delhi, Zoonosis Division National Institute of Communicable Diseases, 2006 (http://www.whoindia.org/LinkFiles/Communi cable_Diseases_Guidelines_for_Prevention_and_Control_ Leptospirosis.pdf, accessed 16 June 2012 ).

11. Raj A. Gender equity and universal health coverage in India. The Lancet, 2011, 377(9766):617-618. doi:10.1016/S01406736(10)62112-5

12. Jena AB, Mohanty KC, Devadasan N. An outbreak of leptospirosis in Orissa, India: the importance of surveillance. Tropical Medicine \& International Health, 2004, 9:1016-1021. doi:10.1111/ j.1365-3156.2004.01293.x pmid:15361116

13. Bayram $\mathrm{C}$ et al. Male consultations in general practice in Australia 1999-2000. General Practice Statistics and Classification Unit, A joint report by the University of Sydney and the Australian Institute of Health and Welfare, AlHW Cat. No. GEP 11, 2003 (http://www. aihw.gov.au/WorkArea/DownloadAsset. aspx?id =6442456030, accessed 19 June 2012). 
